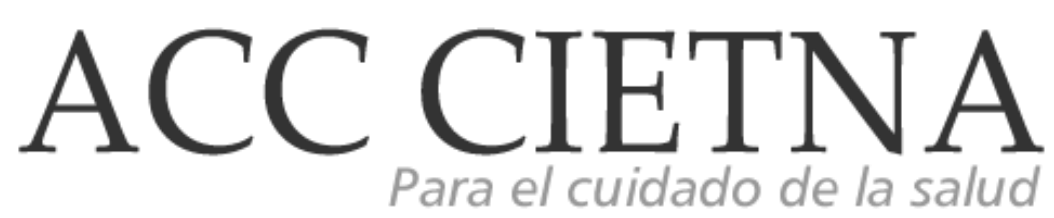

https:// doi.org/10.35383/cietna.v8i1 1.569

ENSAYO

\title{
Comunicación entre padres e hijos adolescentes sobre conductas de riesgo para la salud
}

\author{
Álvarez Aguirre Alicia', Hernández Rodríguez Verónica Margarita²
}

\begin{tabular}{ll}
\hline INFORMACIÓN DEL ARTÍCULO & RESUMEN \\
\hline $\begin{array}{l}\text { Historia del artículo: } \\
\text { Recibido el } 22 \text { de marzo de 2021 }\end{array}$ & Hoy día el adolescente vive sus relaciones interpersonales mediadas \\
Aceptado el 22 de junio de 2021 & por las redes sociales y conducidas socialmente hacia la toma de \\
\hline $\begin{array}{l}\text { Palabras clave: } \\
\text { Padres }\end{array}$ & conductas de riesgo, motivadas desde el poder y la aceptación por su \\
Adolescencia & entorno de pares. Esta circunstancia pone en relieve la importancia que \\
Asertividad & tienen los padres, como esas personas significativas que habrán de \\
& introyectar en el adolescente valores y conductas que impulsen su \\
& desarrollo biopsicosocial. De ahí que, la comunicación entre padres e \\
& hijos es pieza clave y fundamental, ya que el diálogo asertivo y afectivo \\
& facilitará el intercambio de experiencias que serán de utilidad al \\
& adolescente al momento de tomar decisiones. El detalle ante esto, es \\
& que evidencias académicas y científicas apuntan a que dicha \\
& comunicación no se lleva a cabo, dejando al adolescente desprovisto \\
& de elementos que contribuirían a su crecimiento y desarrollo personal. \\
& Por lo que en el siguiente ensayo se despliega una reflexión sobre la \\
& comunicación entre padres e hijos adolescentes respecto a las \\
& conductas de riesgo a la salud.
\end{tabular}

Communication between parents and adolescent children on health behaviors

ABSTRACT

Keywords:

Parents

Adolescence

Communication
Today, the adolescent lives its interpersonal relationships mediated by social networks and socially conducted towards the taking of risk behaviors, motivated from power and acceptance by their peer

'Doctora en Ciencias de Enfermería. Docente del Departamento de Enfermería Clínica, División de Ciencias de la Salud e Ingenierías, Campus Celaya Salvatierra, Universidad de Guanajuato, México. Email: alicia.alvarez@ugto.mx. ORCID: https://orcid.org/0000-0001-5538-7634

2Doctora en Ciencias de Enfermería. Docente de la Facultad de Enfermería, Universidad Autónoma de Querétaro, México. Email: v.hernandez@uaq.mx. ORCID: https://orcid.org/0000-0002-5795-0483 
environment. This circumstance highlights the importance of parents, such as these significant people who will have to introduce in adolescent values and behaviors that promote their biopsychosocial development. Hence, communication between parents and children is a key and fundamental part, since assertive and affective dialogue will facilitate the exchange of experiences that will be useful to the adolescent when making decisions. The detail before this, is that academic and scientific evidence that this communication is not carried out, leaving the adolescent devoid of elements that would contribute to the growth and personal development of it. So, in the following essay, a reflection on communication between parents and adolescent children is deployed regarding health behaviors.

\section{Introducción}

El análisis de las estadísticas de morbi-mortalidad permite observar un repunte en el consumo de sustancias, abuso sexual, depresión y suicidio, problemas de salud pública que en muchas ocasiones son provocados por la comunidad adolescente'. Este aumento de conductas de riesgo en su mayoría es motivado por el grupo de pares y el contexto sociocultural donde se desenvuelve el adolescente ${ }^{2}$. Sin embargo, es necesario reconocer que falta investigación que profundice en los diversos factores que confluyen en este tipo de comportamientos.

El valor que tiene la familia en el interior del adolescente se reconoce en diversos estudios, siendo catalogado como factor protector o factor de riesgo ${ }^{1,3}$, esta ambivalencia, centra el análisis del presente ensayo, en la comunicación entre padres e hijos adolescentes.

A través del diálogo claro y efectivo se pueden prevenir conductas de riesgo a la salud ${ }^{4,6}$. La comunicación asertiva entre padres e hijos adolescentes, propicia la salud de los hijos, porqué al experimentar afecto, confianza y cercanía es menos probable que se involucren en conductas de riesgo. Los adolescentes, dada la etapa en la que se encuentran, se exponen a conductas de riesgo como los accidentes, agresiones, discusiones o riñas, trastornos de la alimentación, conducir con exceso de velocidad o bajo el influjo de alcohol y otras sustancias, adicciones, relaciones sexuales sin protección, embarazo, abuso sexual, además de experimentar sentimientos de tristeza, temor y miedo e intensión de privarse de la vida2,5,7, principalmente en aquellos que viven en condiciones de alta vulnerabilidad. Aunado a que los adolescentes en esta etapa de su vida, que se caracteriza por vivenciar los cambios psicológicos, físicos y sociales, requieren de apoyo de sus padres para afrontar las situaciones del día a día. Por lo anterior es que debe entablarse una comunicación entre padres e hijos adolescentes, que permeé la relación, y así los padres al formar a sus hijos; brinden amor, cuidado, seguridad, confianza, valores, entre otros atributos, que permiten establecer relaciones asertivas con otros y disminuir las conductas de riesgo a la salud. Para lo cual la comunicación deberá ser asertiva, fomentada a través del diálogo abierto y directo entre ambos.

Científicamente se ha comprobado que la comunicación entre padres e hijos adolescentes se asocia positiva y significativamente con la prevención de conducta de riesgo a la salud ${ }^{8,9}$.Sin embargo, aún hoy día, pocos padres hablan sobre estos temas con sus hijos, debido a la falta de conocimientos, desaprobación y vergüenza que les genera tratar estos temas, y al no sentirse 
seguros, expertos y autosuficientes para abordarlos, dejando esta actividad a las instituciones de educación y salud; pese a que los hijos expresan que prefieren hablar con sus padres de estos temas $6,10,11$, que en su etapa de vida cobran mucha importancia. Esta ausencia o limitación de comunicación entre padres e hijos adolescentes se ve influida por la cultura, creencias y valores que caracterizan el medio ambiente familiar5,12. Además que las conductas de riesgo a la salud en los hijos son afectadas por la estructura, cohesión y control parental, pues las actitudes y valores acerca de la sexualidad y las adicciones se fomentan en el hogar 3,13 .

Ante lo mencionado surge la pregunta: ¿Cómo es la comunicación entre padres e hijos adolescentes respecto a las conductas de riesgo a la salud?

En consecuencia, el objetivo del presente ensayo es describir la comunicación entre padres e hijos adolescentes respecto a las conductas de riesgo a la salud.

\section{Desarrollo}

La adolescencia es una etapa donde las estructuras cerebrales aún se encuentran en desarrollo, por lo que todavía son inmaduras, circunstancia que se observa en su comportamiento, así como en sus procesos mentales; factores que los posiciona inherentemente como población vulnerable a conductas de riesgo. A esto se suma que los adolescentes de la época actual nacieron durante el cambio de milenio, donde la tecnología y redes sociales marcan en buena medida las relaciones interpersonales y por ende de comunicación ${ }^{1}$, que estos establecerán con su entorno.

En la última década, se han producido cambios en el estilo de vida de los adolescentes, los cuales se caracterizan por la puesta en marcha de aquellos valores que prevalecen hoy día en la sociedad ${ }^{2}$, como son mayor desinhibición y desenfreno, empujando a los adolescentes a vivir conductas de riesgo a la salud tales como conducta sexual de riesgo y adicciones, en estas situaciones prevalece la ausencia de comunicación entre padres e hijos adolescentes $^{14}$ aunado a la permisividad del consumo de alcohol por los padres y a que la práctica sexual se presenta confusa, contradictoria y con objeto de controversia ${ }^{15}$.

Estas conductas de riesgo impactan directamente en el adolescente que la realiza, pero también genera estragos en aquellos de su entorno (familia, grupo de pares, compañeros de escuela y/o trabajo) $)^{2}$.

La familia y en particular los padres son quienes proveen a los hijos de las herramientas que les permitirán interrelacionarse con otros, y adaptarse a las normas de los distintos contextos donde interactúe 3 .

A pesar de esto, en los últimos años la evidencia empírica, reporta que los padres no hablan con sus hijos sobre temas de sexualidad y adicciones pues piensan que al hacerlo los están orillando a experimentarlo y ven este tipo de conversaciones como una falta de respeto entre ellos ${ }^{16}$; asimismo se señala que las madres con niños de 10 y 11 años, abordan menos temas que aquellas que tienen hijos mayores de 12 años ${ }^{17}$, no obstante, se debe considerar hablar de estos temas a menor edad, porqué las generaciones de hoy en día tienden a iniciar vida sexual y consumo de drogas a edades cada vez más tempranas.

En el núcleo familiar, las madres presentan mayor seguridad para hablar con sus hijos sobre sexualidad y adicciones que los padres ${ }^{18}$, y son las madres las encargadas de platicar con las hijas y los padres a los hijos, especialmente sobre retrasar el inicio de vida sexual, el uso de métodos anticonceptivos y consumir responsablemente bebidas alcohólicas ${ }^{17}$.

Para las autoras los padres tienen el compromiso de abordar abierta y libremente, escuchar y comprender las necesidades y sentimientos de sus hijos, para que estos tengan seguridad y confianza al expresar sus inquietudes y dudas 
sobre lo que están viviendo ${ }^{19}$. Sobre esto algunos de los padres, toman estas conversaciones sin problema: "hablar de estos temas, es algo que estaba destinado para una determinada edad" 16 . Comentan que es necesario e importante llevar a cabo conversaciones con sus hijos, pues al hablar con ellos sobre temas de conductas de riesgo a la salud, también los hijos sentirán la confianza para hablar sobre cualquier situación por la que estén atravesando20. Aquellos hijos que tienen una comunicación efectiva con sus padres presentan mayor autoestima y motivación, por lo tanto, retrasan las conductas de riesgo a la salud o en su caso favorecen el involucrarse en conductas responsables y seguras ${ }^{19}$.

Por lo anterior, la comunicación asertiva es un protector de las conductas de riesgo a la salud, que de cierta manera ejerce un control que beneficia el autocuidado y bienestar de los adolescentes, los estudios han demostrado que el manejo de las situaciones de riesgo que presentan los adolescentes se relacionan con diferentes habilidades protectoras, destacando la comunicación asertiva ${ }^{4,21}$. Por consiguiente, los padres deben prepararse para desarrollar su capacidad de comunicación asertiva con sus hijos en especial con los adolescentes ya que es una etapa de aprendizaje.

La comunicación asertiva, se considera un estilo de comunicación efectiva y satisfactoria, cuyos elementos básicos son: respetarse a sí mismo, respetar a los demás, ser directo, honesto, positivo y oportuno y tener control emocional. En la comunicación asertiva se tiene el contenido del mensaje (sentimientos, derechos, opiniones, solicitudes y límites) así como el estilo no verbal del mensaje (contacto visual, postura y expresión facial) $^{22}$. Los rasgos de una persona que posee una comunicación asertiva incluyen: a) El sentirse libre para manifestarse mediante palabras y actos; b) Puede comunicarse con personas extrañas, familiares y amigos; c) Su comunicación es siempre abierta, directa, franca y adecuada, d)
Tiene una meta en la vida; e) Actúa de una forma respetable ${ }^{23}$.

En este sentido la comunicación asertiva se entiende como la manifestación clara y directa de lo que los individuos desean y no desean, cuando interactúan con otras personas. Por tanto, una comunicación asertiva permite expresar sentimientos, opiniones, intenciones, posturas, creencias y pensamientos, a través de conducta verbal y no verbal, en el momento y lugar oportuno, de forma pertinente y sin negar los derechos de los demás. La comunicación asertiva permite hacer llegar a los demás los propios mensajes, genera sentimientos de seguridad, autoestima y reconocimiento, es una habilidad aprendida mediante el entrenamiento ${ }^{24}$. Este entrenamiento se caracteriza como una combinación de técnicas conductuales para resolver problemas interpersonales, $\forall$ que permite reducir la respuesta de ansiedad que representa el hablar y promover una comunicación más efectiva con otros 25,26 .

Para estas autoras los padres con comunicación asertiva tienen la capacidad de comunicarse con seguridad, expresan sus sentimientos, creencias y opiniones con honestidad sobre sexualidad y adicciones sin tener que recurrir a comportamientos pasivos, agresivos 0 manipuladores. El entrenamiento de los padres para adquirir la comunicación asertiva consiste en reducir la ansiedad que genera hablar con sus hijos adolescentes sobre temas de sexualidad y adicciones, para ello se sugiere fortalecer la confianza de los padres para entablar la comunicación respecto de estos temas.

En el entrenamiento asertivo como intervención de enfermería ${ }^{27}$ se sugiere que los profesionales de enfermería realicen las siguientes actividades: a) Identificar y reducir las barreras para la comunicación asertiva; b) Diferenciar entre conductas asertivas, agresivas, pasivas y manipuladoras; c) Promover la expresión de pensamientos y sentimientos, tanto positivos como negativos; d) Enseñar sobre estrategias para 
la práctica de una comunicación asertiva p. ej., a través de la modelación, discusión y juego de roles los padres pueden aprender a realizar solicitudes, responder que no a solicitudes no razonables, e iniciar y finalizar una conversación sobre temas de sexualidad y adicciones; estas herramientas permitirán al padre articular con precisión la información sobre estos temas con sus hijos adolescentes.

\section{Conclusiones}

Se puede concluir que hoy día hay poca o nula comunicación entre padres e hijos adolescentes, y en su mayoría se ha dejado a la madre esa responsabilidad, salvo en los temas de adicciones donde ambos padres comparten esa tarea. Por otro lado, la comunicación asertiva entre padres e hijos adolescentes ha demostrado tener resultados positivos respecto a que contribuye a la autonomía e identidad que busca conseguir el adolescente, lo que a la larga promoverá el desarrollo de adultos con conductas saludables a nivel físico, psicológico y social. Empero para esto, es necesario suscitar ese diálogo entre ambos actores, para lo cual se hace necesario ayudar a los padres a gestar en sí mismos esa seguridad y autoconfianza para el abordaje de temas que potencialmente les generan estrés o ansiedad. Para que finalmente se atrevan y se den la oportunidad de entablar conversaciones con sus hijos, de tal forma que los adolescentes logren percibir esa confianza, afecto y amor, a fin de que se apropien de la información asertiva que se le está proporcionando por parte de sus padres.

\section{Bibliografía}

1. Palacios X. Adolescencia: ¿una etapa problemática del desarrollo humano? Revista Ciencias de la Salud [Internet]. 2019 [Consultado 10 Mar 2021]; 17(1): 5-7. Disponible en https://dialnet.unirioja.es/servlet/articulo?c odigo $=6844569$

2. Salas F. Caracterización de factores implicados en las conductas de riesgo en adolescentes. ABRA [Internet]. 2018 [Consultado 10 Mar 2021]; 38(56): 1-6. Disponible en: https://doi.org/ 10.15359 /abra.38-56.3

3. Álvarez-Trujillo E, Cornelio-Landero R, Macías-Murguía G. Alternativas de intervención en conductas de riesgo en estudiantes de bachillerato. Revista Innova Educación. 2020 [Consultado 10 Mar 2021]; 2(4): 638-657. Disponible en: https://www.revistainnovaeducacion.com/in dex.php/rie/article/view/93

4. Bandura A. Como afrontamos los cambios de la sociedad actual. España: Desclee de Brouwer; 1999. 279p.

5. Prado G, Pantin H, Huang S, Cordova D, Tapia MI, Velazquez MR, et al. Effects of a Family Intervention in Reducing HIV Risk Behaviors Among High-Risk Hispanic Adolescents. Arch Pediatr Adolesc Med [Internet]. 2012 [Consultado 12 Mar 2021]; 166(2): 127-33. Disponible en: https://doi.org/10.1001/archpediatrics.201 1.189

6. Mosquera J, Mateus J. Conocimientos, actitudes y prácticas sobre métodos de planificación familiar, VIH/SIDA y el uso de los medios de comunicación en jóvenes. Colombia médica [Internet]. 2003 [Consultado 12 Mar 2021]; 34(4): 206-12. Disponible en https://colombiamedica.univalle.edu.co/ind ex.php/comedica/article/view/275

7. McKay M, Alicea S, Elwyn L, McClain Z, Parker G, Small L, et al. The Development and Implementation of Theory-Driven Programs Capable of Addressing Poverty-Impacted Children's Health, Mental Health, and Prevention Needs: CHAMP and CHAMP+, evidence-Informed, Family-Based Interventions to Address HIV Risk and Care. Journal of Clinical Child and adolescent psychology, the official journal for the Society of Clinical Child and Adolescent Psychology, American Psychological Association, Division 53 [Internet]. 2014 [Consultado 12 Mar 
2021]; 43(3): 428-41. Disponible en: https://doi.org/10.1080/15374416.2014.8 93519

8. Osorio-Leyva A, Álvarez-Aguirre A, Hernández-Rodríguez V, Sánchez-Perales $M$, Muñoz-Alonso L. Relación entre asertividad sexual y autoeficacia para prevenir el $\mathrm{VIH} / \mathrm{SIDA}$ en jóvenes universitarios del área de la salud. RIDE. Rev. Iberoam. Investig. Desarro. Educ [Internet]. 2017 [Consultado 12 Mar 2021]; 7(14): 1-14. Disponible en: https://doi.org/10.23913/ride.v7i14.264

9. Fernández-Tapia $S$. Determinantes sociales que condicionan la actividad sexual precoz de los adolescentes. Revista Peruana de Ciencias de la Salud [Internet]. 2020 [Consultado 14 Mar 2021]; 2(3):161-9. Disponible en: https://doi.org/10.37711/rpcs.2020.2.3.19 4

10. Friedman, M. Family nursing. Theory and assessment. Introduction to the family, The United States of America: Appleton Century Crofts; 1981.

11. Bárcena-Gaona S, Guevara-Benítez $Y$, Rodríguez-Gutiérrez $M$. Programa de intervención para promover la comunicación sobre sexualidad en padres de hijos con discapacidad intelectual. Psicología y salud [Internet]. 2020 [Consultado 14 Mar 2021]; 30(2): 173-87. Disponible en: https://doi.org/10.25009/pys.v30i2.2652

12. Kusheta S, Bancha B, Habtu Y, Helamo D, Yohannes S. Adolescent-parent communication on sexual and reproductive health issues and its factors among secondary and preparatory school students in Hadiya Zone, Southern Ethiopia: institution based cross sectional study. BMC Pediatr [Internet]. 2019 [Consultado 14 Mar 2021];19 (9): 2-11. Disponible en: https://doi.org/10.1186/s12887-0181388-0

13. Koren A. Reproductive Health for Teens: Parents Want In Too, Journal of Sex \& Marital Therapy [Internet]. 2019 [Consultado $18 \mathrm{Mar}$ 2021]; 45(5): 406-13. Disponible en:
https://doi.org/1080/0092623X.2018.1549 $\underline{635}$

14. Palacios J, Álvarez M. Consumo de drogas asociadas al contagio de infecciones de trasmisión sexual en jóvenes de México. Health and Addictions [Internet]. 2018 [Consultado 14 Mar 2021]; 18(2): 111-20. Disponible en: https://doi.org/ 10.21134 /haaj.v1 8i2.384

15. Liu T, Fuller J, Hutton A, Grant J. Factors shaping parent-adolescent communication about sexuality in urban China, Sex Education [Internet]. 2017 [Consultado 18 Mar 2021]; 17 (2): 180-94. Disponible en: https://doi.org/10.1080/14681811.2016.1 $\underline{276897}$

16. Bangpan M, Operario D. Understanding the role of family on sexual-risk decisions of young women: A systematic review. AIDS Care [Internet]. 2012 [Consultado 18 Mar 2021]; 24(9): 2-10. Disponible en: https://doi.org/10.1080/09540121.2012.6 99667

17. Rouvier M, Campero L, Walker D, Caballero M. Factors that influence communication about sexuality between parents and adolescents in the cultural context of Mexican families. Sex Education [Internet]. 2011 [Consultado 18 Mar 2021]; 11(2): 175-91. Disponible en: https://eric.ed.gov/?id=EJ925059

18. Wilson E, Koo H. Mothers, fathers, sons, and daugthers: gender differences in factors associated with parent-child communication about sexual topics. Reproductive Health [Internet]. 2010 [Consultado 18 Mar 2021]; 7(31): 1-9. Disponible en: https://doi.org/10.1186/1742-4755-7-31

19. Kuo C, Atujuna M, Mathews C, Stein D, Hoare $J$, Beardslee W, et al. Developing family interventions for adolescent HIV prevention in South Africa. Aids Care [Internet]. 2016 [Consultado 18 Mar 2021]; 28(1): 1-4. Disponible en: https://doi.org/10.1080/09540121.2016.1 146396 
20. Vig J, Miller K, Chirwa-Motswere C, Winskell $\mathrm{K}$, Stallcup E. Involving parents from the start: Formative evaluation for a large RCT with Botswana Junior Secondary School students. HHS Public [Internet]. 2015 [Consultado 18 Mar 2021]; 15(1): 9-15. Disponible en: https://doi.org/10.2989/16085906.2015.1 135295

21. Smith M, Liehr P. Middle range theory for nursing. NY: SpringerPublishing Company; 2018.

22. Alberti R, Emmons $M$. Viviendo con autoestima: como fortalecer con asertividad lo mejor de tu persona. México: Pax; 1990.

23. Fensterheim $\mathrm{H}$, Baer J. No diga Si cuando quiera decir No. Barcelona: Grijalbo; 1975.

24. Rathus S. A 30-item schedule for assessing assertive behavior. Behavior Therapy [Internet]. 1973 [Consultado 18 Mar 2021]; 4(3): 398-406. Disponible en: https://doi.org/10.1016/S00057894(73)80120-0

25. Aduna A, Bolaños J. Curso para reducir la ansiedad ante la participación en grupo (escolar y social). México: Editorial Trillas; 1996.

26. Cooley M. Interests of assertivenss trainees. Journal of Counseling Psychology [Internet]. 1979 [Consultado 18 Mar 2021]; 26(2):173$175 . \quad$ Disponible en: https://doi.org/10.1037/0022$\underline{0167.26 .2 .173}$

27. Rifá R, Olivé C, Lamoglia M. Lenguaje NIC para el aprendizaje teórico-práctico en enfermería. España: ELSEVIER; 2020 